Article

\title{
Interior of Amylopectin and Nano-Sized Amylopectin Fragments Probed by Viscometry, Dynamic Light Scattering, and Pyrene Excimer Fluorescence
}

\author{
Lu Li and Jean Duhamel * \\ Department of Chemistry, Institute for Polymer Research, Waterloo Institute for Nanotechnology, \\ University of Waterloo, Waterloo, ON N2L 3G1, Canada; 183li@uwaterloo.ca \\ * Correspondence: jduhamel@uwaterloo.ca
}

Received: 23 October 2020; Accepted: 8 November 2020; Published: 11 November 2020

\begin{abstract}
Nano-sized amylopectin fragments (NAFs), prepared by extrusion of waxy corn starch, were investigated by viscometry, dynamic light scattering (DLS), and pyrene excimer fluorescence (PEF). NAF57, with a hydrodynamic diameter of $57 \mathrm{~nm}$, was treated with nitric acid to yield three degraded NAFs, which appeared to share the same interior and structural features as amylopectin based on their measured intrinsic viscosity and hydrodynamic diameter. This conclusion was further supported by comparing the efficiency of forming excimer between an excited and a ground-state pyrenyl label covalently attached to the NAFs (Py-NAFs) using their $I_{\mathrm{E}} / I_{\mathrm{M}}$ ratio of the fluorescence intensity of the excimer $\left(I_{\mathrm{E}}\right)$ to that of the monomer $\left(I_{\mathrm{M}}\right)$. The overlapping trends obtained for all Py-NAFs and the pyrene-labeled amylopectin samples by plotting the $I_{\mathrm{E}} / I_{\mathrm{M}}$ ratio as a function of pyrene content provided further evidence that the interior of NAFs and amylopectin shared the same structural features and contained a similar amount of free volume as predicted by the Solution-Cluster (Sol-CL) model. The presence of free volume was validated by adding linear poly(ethylene glycol) (PEG) chains that could not penetrate the interior of Py-NAFs, thus subjecting the Py-NAFs to increased osmotic pressure, which induced their compression and resulted in an increase in $I_{\mathrm{E}} / I_{\mathrm{M}}$.
\end{abstract}

Keywords: amylopectin; pyrene excimer fluorescence; nano-sized amylopectin fragments

\section{Introduction}

Starch nanoparticles (SNPs) can be employed as substitutes of petroleum-based latex used in the paper coating industry, and their potential as food additives or drug carriers is being actively researched [1]. SNPs are expected to retain the main advantages of starch, which is abundant, cost-effective, non-toxic, and renewable [2]. A number of different SNPs have been generated by chemical, biochemical, or mechanical processes. Examples of such processes include acid or enzymatic hydrolysis, high pressure homogenization, ultrasonication, or reactive extrusion [1]. One of the goals of these processes is to reduce the size of starch to increase the dispersibility of the particles in solution and enable the adjustment of their size to meet the requirements for different industry applications [3]. Unfortunately, the properties of starch and SNPs are somewhat limited and they do not meet the vast majority of applications relevant to the polymer industry [4-6]. To expand the applicability of SNPs, the chemical modification of SNPs is currently the object of intense research. Most chemical modifications have aimed so far at imparting some hydrophobicity to otherwise hydrophilic SNPs by attaching onto them octenyl succinic anhydride [7,8], oleic and stearic acids [9], polystyrene [10], cholesterol [11], and, more recently, propionic and hexanoic anhydride [12,13]. However, to be most effective, these chemical modifications require the characterization of SNPs not only from a viewpoint pertaining to synthetic polymer particles, based on their hydrodynamic diameter $\left(D_{\mathrm{h}}\right)$ 
and internal density determined from, respectively, dynamic light scattering (DLS) and intrinsic viscosity $([\eta])$ measurements, but also, perhaps more importantly, as biopolymer particles, open entities whose interior must be understood at the molecular level in solvents, where they can form a nanodispersion [8]. Such studies are particularly relevant for non-crystalline SNPs such as those produced through extrusion, whereby the crystalline microdomains of the starch granules are fully melted at the high temperatures experienced in the extruder [14]. Upon dispersion in a suitable solvent like dimethyl sulfoxide (DMSO) or water, such SNPs are swollen and their interior, which is exposed to the solvent, can readily react with the chemicals desired for a given chemical modification. A recent study has shown that SNPs produced through extrusion behave differently from typical synthetic polymer particles and that the term particle might be inadequate to describe SNPs [12]. Instead, it was suggested that SNPs could be described as nano-sized starch fragments (NSFs) [12]. The nature of NSFs is still poorly understood and their interior would be worthy of more detailed investigation.

The Solution-Cluster (Sol-CL) model could help in this regard. It was recently introduced to describe the spatial arrangement of the structural motives of amylopectin dispersed in DMSO [15]. It suggests that amylopectin is constituted of dense clusters of oligosaccharide single helices, which are separated from one another by longer linear oligosaccharides. In this representation, the clusters of oligosaccharide helices represent the building blocks of amylopectin. The clusters of helices are compact, whereas the longer oligosaccharides linking the clusters to one another generate free volume in the amylopectin interior. A larger amylopectin macromolecule should thus have more clusters of helices and more linear oligosaccharide segments, which would generate more free volume. These concepts are illustrated in Figure 1, which shows the spatial arrangement of clusters of helices in amylopectin samples of different sizes.

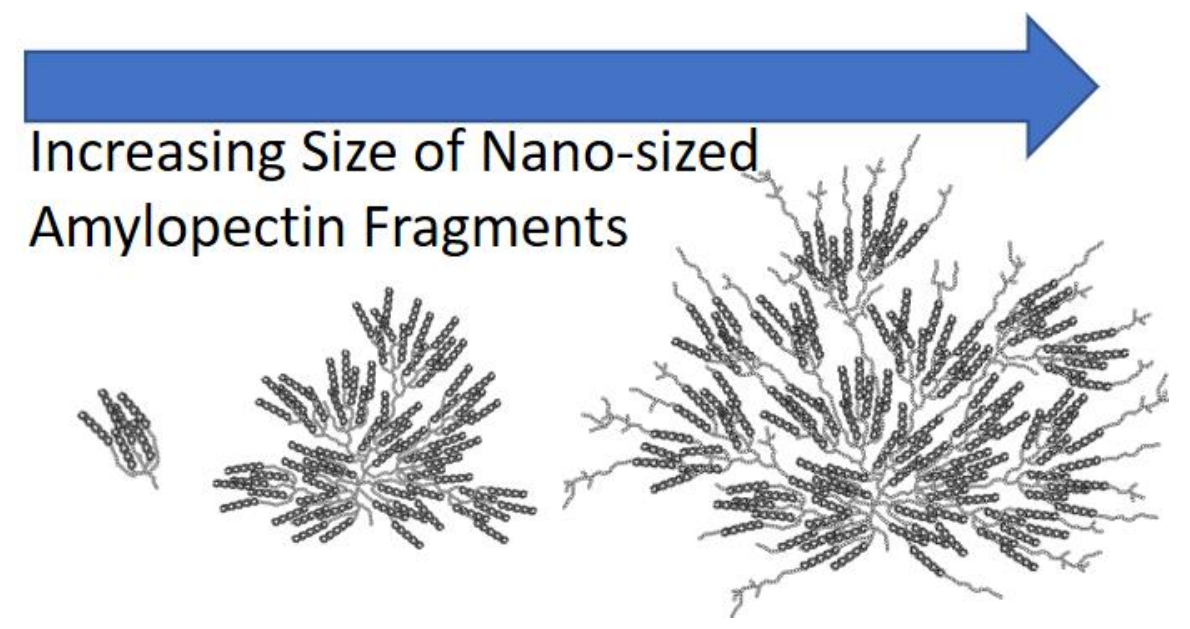

Figure 1. Depiction of the spatial arrangement of the clusters of helices for amylopectin samples of different sizes as predicted by the Solution-Cluster (Sol-CL) model [15].

Based on the Sol-CL model, the smaller nano-sized amylopectin fragments (NAFs) produced from amylopectin should have less excluded volume, making them more rigid and less malleable objects in solution. The study of this effect requires one to measure the size of NAFs and their internal density, as the NAFs are being compressed. To this end, dynamic light scattering (DLS), intrinsic viscosity $([\eta])$, and pyrene excimer fluorescence/formation (PEF) were used to determine the hydrodynamic diameter $\left(D_{\mathrm{h}}\right)$ of the NAFs, measure their overall density when dispersed in DMSO, and probe the local density experienced by the oligosaccharide side chains constituting the NAFs, respectively. These experiments took advantage of the difference in the length scales probed by each technique. While DLS and viscometry probe macromolecules in their entirety over length scales, which ranged from 8.3 to several hundreds of nanometers for the NAFs and amylopectin samples studied, PEF probes macromolecules over a length scale of $4.6 \mathrm{~nm}$ or less, when the dye 1-pyrenebutyric acid (PyBA) is 
used to label polysaccharides at their C2-hydroxyl [15]. Furthermore, the process of excimer formation, where an excimer is the product of the encounter between an excited and a ground-state pyrene, can be quantified by determining the ratio $I_{\mathrm{E}} / I_{\mathrm{M}}$ of the fluorescence intensity of the excimer $\left(I_{\mathrm{E}}\right)$ over that of the monomer $\left(I_{\mathrm{M}}\right)[16]$. In turn, the $I_{\mathrm{E}} / I_{\mathrm{M}}$ ratio depends directly on the local concentration of pyrenyl labels ([Py $]_{\mathrm{loc}}$ ) covalently attached onto a macromolecule [17]. Changes in the $I_{\mathrm{E}} / I_{\mathrm{M}}$ ratio of a polysaccharide fluorescently labeled with PyBA indicate changes in $[P y]_{l o c}$, and thus in the local concentration of the oligosaccharide side chains onto which PyBA is covalently attached.

These concepts were implemented in the present study that provides additional evidence in support of the Sol-CL model introduced earlier [15] and affords a better understanding of the arrangement of oligosaccharide side chains inside NAFs. The experiments described in this paper used the same 21 research-grade NAFs and one amylopectin sample from waxy maize whose characterization has already been reported [15]. In particular, it introduces new experiments, where the degradation of a large NAF by nitric acid was found to yield smaller NAFs, whose $D_{\mathrm{h}}$ and $[\eta]$ matched the values expected of smaller NAFs. This suggested that the degraded NAFs retained the same arrangement of oligosaccharide side chains in the particle interior as that of the original NAF they were produced from, in the same manner as the NAFs retained the same arrangement of oligosaccharide side chains in their interior as amylopectin from which they were generated. Further support for the common interior shared by NAFs and amylopectin was obtained from PEF measurements which showed that the $I_{\mathrm{E}} / I_{\mathrm{M}}$ ratios obtained for dispersions in DMSO of Py-Amylopectin, Py-NAF57, Py-NAP20, and Py-NAF8.3, with $D_{\mathrm{h}}$ values of 225, 57, 20, and $8.3 \mathrm{~nm}$, respectively, clustered around a single master line. This behavior demonstrated that, on the $\sim 4.6 \mathrm{~nm}$ length scale for PEF, these four polysaccharides shared a same interior regardless of the overall size of the polysaccharide. The main difference between the polysaccharides was the amount of excluded volume that was expected to increase with increasing polysaccharide size as predicted by the Sol-CL model.

The difference in excluded volume present in the Py-NAFs was further examined by probing the deformability of Py-NAFs in dilute dispersions by increasing the osmotic pressure experienced by the Py-NAFs upon addition of poly(ethylene glycol)s (PEGs) with molecular weights of $0.2,0.4$, 2.0, 5.0, and $10 \mathrm{~K}$ to the polysaccharide dispersions in DMSO. On the one hand, the smaller PEGs diffused throughout the Py-NAF interior, and as the solution viscosity increased with increasing PEG concentration, diffusive encounters between pyrenyl labels were reduced and PEF decreased. The larger PEGs, on the other hand, were unable to penetrate the Py-NAF interior, which increased the osmotic pressure experienced by the Py-NAFs as the PEG concentration was increased. Compression of the Py-NAFs raised the local pyrene concentration, which favored PEF. The deformability experienced by the Py-NAFs under increased osmotic pressure was taken as evidence for the existence of excluded volume in the NAF interior. However, the deformability of the Py-NAFs was strongly reduced for smaller NAFs as smaller NAFs exhibited less free volume.

Consequently, this study on Py-NAFs confirmed the existence of oligosaccharide-rich clusters inside NAFs separated by the excluded volume generated by linear oligosaccharide segments bridging the clusters of helices. These considerations are a consequence of the Sol-CL model and are illustrated in Figure 1. This study represents another example of the use of PEF in the characterization of polysaccharides in solution.

\section{Experimental Section}

\subsection{Instrumentation}

The instruments used in this study include an Innova 4000 incubator shaker (NewBrunswik, Edison, NJ, USA) to prepare the polysaccharide dispersions, a Freezone 6 Labconco (Labconco, Kansas City, MO, USA) freeze-dryer for lyophilization, a Photon Technology International LS-100 (PTI, London, ON, Canada) steady-state fluorometer to acquire the fluorescence emission spectra, a time-resolved fluorometer equipped with an IBH $340 \mathrm{~nm}$ NanoLED (IBH, Glasgow, Scotland, UK) to 
acquire the fluorescence decays, a Zetasizer NanoZS (Malvern, Worcester, WR, UK) for dynamic light scattering (DLS) measurements, a Varian Cary 100 Bio spectrophotometer (Varian, Palo Alto, CA, USA) to acquire UV-VIS absorbance spectra, and a Cannon D449-200 Dilution Ubbelohde viscometer (Cannon Instrument Company, State College, PA, USA) for intrinsic viscosity measurements.

\subsection{Chemicals}

Twenty-one research-grade NAFs were supplied by EcoSynthetix (Burlington, ON, Canada) by extruding waxy corn starch, which is $>99 \%$ amylopectin, under different conditions. Their number average particle hydrodynamic diameter $\left(D_{\mathrm{h}}\right)$ ranging from 8.3 to $57 \mathrm{~nm}$ was measured by DLS [15]. As a precaution to remove possible additives left over during the production of NAFs, all NAFs were purified by precipitation. Doubly distilled Milli-Q water was obtained from a Millipore Milli-RO 10 Plus or Milli-Q UFPlus (Bedford, MA, USA) system. Dialysis tubing with a $1 \mathrm{kDa}$ molecular weight cutoff (MWCO) was purchased from Spectrum Laboratories Inc. (Spectrum Laboratories Inc., Rancho Dominguez, CA, USA).

\subsection{Purification of the NAFs by Precipitation}

Mixtures of NAFs ( $5 \mathrm{wt} \%$ ) in DMSO were placed in a shaker and kept stirring at $250 \mathrm{rpm}$ and $60{ }^{\circ} \mathrm{C}$ overnight to generate homogeneous NAF dispersions. After the dispersion cooled to room temperature, the NAFs were precipitated dropwise into ethanol. Suction filtration of the precipitate yielded the NAFs. They were rinsed 4 times with acetone to remove any trace of leftover DMSO. The NAF product was then dried overnight in a vacuum oven at $40^{\circ} \mathrm{C}$.

\subsection{Dynamic Light Scattering (DLS)}

NAFs were dispersed at a concentration of $1 \mathrm{~g} / \mathrm{L}$ in DMSO by leaving the mixtures overnight in a shaker set at $250 \mathrm{rpm}$ and $60^{\circ} \mathrm{C}$. All DLS measurements were conducted at $25^{\circ} \mathrm{C}$ by acquiring the autocorrelation function of the light scattering signal over $5 \mathrm{~min}$ in order to obtain a stable baseline. Measurements were repeated 4 times to obtain an average of the number average $D_{\mathrm{h}}$ determined by DLS for each sample.

\subsection{Viscometry}

Homogenous NAF dispersions with NAF concentration between 1 and $8 \mathrm{~g} / \mathrm{L}$ in DMSO were obtained by keeping the samples in a shaker set at $250 \mathrm{rpm}$ and $60^{\circ} \mathrm{C}$ overnight. A Cannon Ubbelohde viscometer was used to measure the viscosity of the NAF dispersions in DMSO $\left(\eta=1.99 \mathrm{mPa} \cdot \mathrm{s}\right.$ at $\left.25^{\circ} \mathrm{C}\right)$. The temperature of the viscometer was kept at $25^{\circ} \mathrm{C}$ during the measurements with a circulating water bath.

\subsection{Density of PEO(5.0K) Solutions in DMSO}

Solutions of PEO(5.0K) in DMSO were prepared. A carefully weighed mass of about $5 \mathrm{~g}$ of each $\mathrm{PEO}(5.0 \mathrm{~K})$ solution in DMSO was placed in a cylinder and the volume measured to determine the density of the solution $(d)$. The relationship shown in Equation (1) was established between $d$ and the weight fraction $\left(f_{\mathrm{w}}\right)$ of polymer for $\mathrm{PEO}(5.0 \mathrm{~K})$ concentrations between 0.1 and $41 \mathrm{wt} \%$.

$$
d=1.10+0.328 \times f_{\mathrm{w}}
$$

\subsection{Degradation of NAF57 by Nitric Acid}

The largest NAF, namely NAF57 where the number after NAF indicates its $D_{\mathrm{h}}$, was dispersed in water at a concentration of $10 \mathrm{wt} \%$ at $60^{\circ} \mathrm{C}$. The degradation was initiated after adjusting the solution $\mathrm{pH}$ to 1 by adding nitric acid. After the desired samples were obtained, the degradation was quenched by adjusting the $\mathrm{pH}$ to 6 using a $0.1 \mathrm{M}$ sodium hydroxide aqueous solution. The sodium chloride salt 
was eliminated by dialysis against double distilled Milli-Q water. The degraded NAFs were recovered by lyophilization.

\subsection{Synthesis of Pyrene-Labeled NAFs (Py-NAFs)}

The preparation of all pyrene-labeled constructs used in this study has been described earlier in detail [15].

\subsection{Deformability of the Polysaccharides}

The deformability of Py(4.8)-NAF57 and Py(5.8)-NAF8.3, where the polysaccharide substrate was labeled with 4.8 and $5.8 \mathrm{~mol} \%$ pyrene and the last number is the $D_{\mathrm{h}}$ expressed in nanometer, was investigated. The Py-NAFs were dispersed in DMSO using a pyrene concentration of $2.5 \times 10^{-6} \mathrm{M}$ equivalent to polysaccharide concentrations of 7.8 and $6.3 \mathrm{mg} / \mathrm{L}$ for Py(4.8)-NAF57 and Py(5.8)-NAF8.3, respectively. Py-NAF dispersions with PEG concentrations ranging from 0 to $60 \mathrm{wt} \%$ were prepared by adding PEGs with a molecular weight of $0.2,0.4,2.0,5.0$, and $10 \mathrm{~K}$ to the Py-NAF dispersions. The dispersions were heated to $60{ }^{\circ} \mathrm{C}$ for $30 \mathrm{~min}$ to fully dissolve the PEGs. After the dispersions were equilibrated to room temperature, they were transferred to the fluorescence cell with minimum disturbance to avoid the eventual crystallization of PEGs at high concentration and their steady-state fluorescence spectra were acquired at different PEG concentrations.

\subsection{Fluorescence Blob Model (FBM) Analysis of the Fluorescence Decays of Py(4.8)-NAF57}

The global FBM analysis of the pyrene monomer and excimer fluorescence decays acquired with Py-NAF dispersions in DMSO has been described earlier in detail [15] and are presented in the Supplementary Materials. In brief, the FBM compartmentalizes the Py-NAF macromolecular volume into smaller equivalent subvolumes referred to as blobs, which represent the volume probed by an excited pyrenyl label. Random labeling of the NAFs to yield Py-NAF ensured that the pyrenyl labels were randomly distributed among the blobs according to a Poisson distribution defined by the average number $\langle n\rangle$ of pyrenyl labels per $b l o b$. The FBM analysis of the decays retrieved $\langle n\rangle$, which was then related to the number ( $N_{\text {blob }}$ ) of anhydroglucose units (AGUs) located inside one $b l o b$ according to Equation (2).

$$
N_{\text {blob }}=\frac{1-f_{\text {Mfree }}}{x}\langle n\rangle
$$

In Equation (2), $x$ represents the molar fraction of pyrene-labeled AGUs in a pyrene-labeled polysaccharide and $f_{\text {Mfree }}$ is the molar fraction of pyrenyl labels that do not form excimer obtained from the fit of the pyrene monomer fluorescence decay (see the Supplementary Materials).

\section{Results and Discussion}

Several experiments involving DLS, viscometry, and PEF were conducted to probe the interior of NAFs dispersed in DMSO. The results of these experiments provided further support for the notion that NAFs and amylopectin share a similar interior constituted of compact clusters of oligosaccharide helices held together by long linear oligosaccharide segments, which generate excluded volume in the macromolecular volume.

\subsection{Viscometry and DLS Experiments}

One of the implications of the Sol-CL model is that degradation of amylopectin should generate nano-sized amylopectin fragments (NAFs) with an interior that should reflect that of amylopectin, as suggested in Figure 1. This was indeed observed earlier for a series of 21 research-grade NAFs with different $D_{\mathrm{h}}$ values that were prepared by extruding waxy corn starch, which is $>99 \%$ amylopectin, under different conditions [15]. As described earlier, iodine binding experiments and ${ }^{1} \mathrm{H}$ NMR measurements demonstrated that the NAFs were amylose free and had the same chemical composition 
as the amylopectin, from which they were prepared [15]. When [ $\eta]$ was plotted against $D_{\mathrm{h}}$ for the NAFs, as seen in Figure 2, all data points were found to cluster around a master curve [15] that lined up with those obtained for different samples prepared from amylopectin as described in an earlier publication [18]. Taking $2.5 /[\eta]$ as the density of the polysaccharide particles since it equals $M /\left(V_{h} \times N_{A}\right)$, a substantial decrease in the density of the polysaccharide was observed with increasing $D_{\mathrm{h}}$ of the polysaccharides, as seen in Figure 2. The fact that all [ $\left.\eta\right]$ and $D_{\mathrm{h}}$ values clustered around a same master line in Figure 2 was taken as evidence that the NAFs shared a similar internal architecture, resulting in an internal density given by $2.5 /[\eta]$ that would decrease with increasing $D_{\mathrm{h}}$. The decrease in polysaccharide density with increasing polysaccharide size was viewed as a clear indication that the interior of amylopectin and the NAFs prepared from it had some excluded volume whose contribution increased with increasing polysaccharide size.

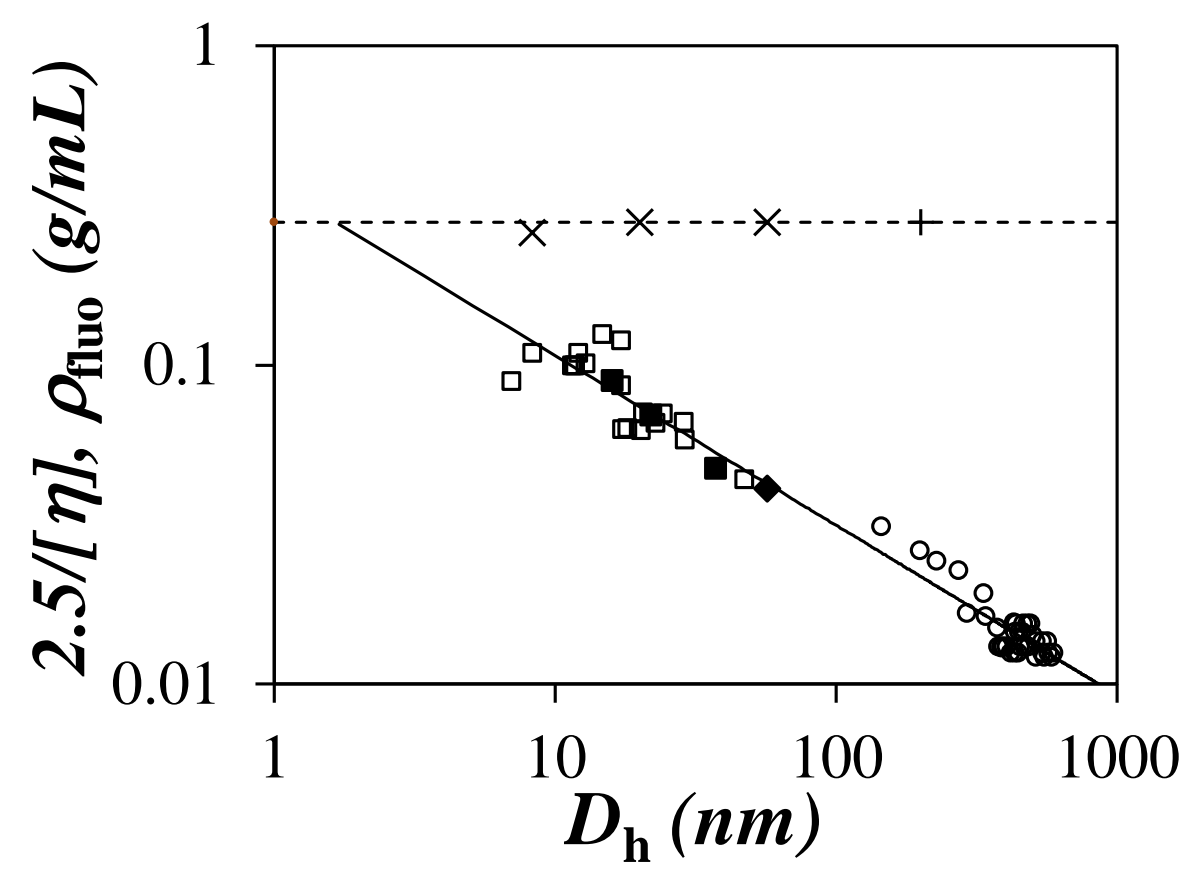

Figure 2. Plot of $2.5 /[\eta]$ obtained by viscometry and $\rho_{\text {luo }}$ obtained by pyrene excimer fluorescence (PEF)

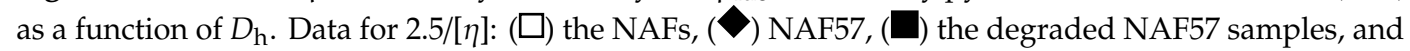
(O) a series of samples derived from amylopectin in dimethyl sulfoxide (DMSO). Data for $\rho_{\text {fluo }}:(\times)$ NAFs and (+) amylopectin [12]. $[\eta]=6.9 \times D_{\mathrm{h}}{ }^{0.53}$.

PEF experiments also determined that the density $\left(\rho_{\text {fluo }}\right)$ experienced by the pyrenyl labels remained constant with the polysaccharide size, as seen in Figure 2, and was one to two orders of magnitude higher than the quantity $2.5 /[\eta]$ [15]. This discrepancy was attributed to the difference in length scales probed by fluorescence and viscometry, with fluorescence probing solely the denser regions corresponding to the clusters of helices in the interior of the polysaccharides, whereas $2.5 /[\eta]$ represented the density of the entire polysaccharides.

Interestingly, as seen in Figure 2, the master line obtained for the NAFs generated through the breakdown of amylopectin induced by the extrusion of waxy corn starch could be reproduced by taking the largest sample among the 21 NAFs that had a $D_{\mathrm{h}}$ of $57 \mathrm{~nm}$ (NAF57) and subjecting it to a nitric acid treatment. Application of the nitric acid treatment to NAF57 over different reaction times yielded three degraded NAFs. As shown in Figure 2, the degraded NAF57 products also clustered along the same master curve as the other NAFs. This result was taken as evidence that the variation in particle density with size shown in Figure 2 was due to the highly branched nature of amylopectin, from which all these NAFs were created and which was reproduced in all the NAFs but on a smaller length scale, as suggested in Figure 1. Interestingly, it also suggested that nitric acid targeted the 
loose linear segments connecting the clusters of helices for degradation, whereas the more compact clusters of helices might have provided some protection against chemical degradation by making the polysaccharide backbone less accessible to nitric acid. As a result, the nitric acid treatment generated smaller NAFs that retained the same internal structure as their parent amylopectin molecule. A better experimental design would have used amylopectin as the starting material for degradation. However, the amylopectin sample used in the study had a broad size distribution, making its characterization by DLS difficult and, thus, the determination of its $D_{\mathrm{h}}$ challenging. Instead, the $[\eta]$ value of $122 \mathrm{~mL} / \mathrm{g}$ determined experimentally for the amylopectin sample in DMSO was used with the master curve in Figure 1 to determine that it had a $D_{\mathrm{h}}$ of $225 \mathrm{~nm}$. Its degradation with nitric acid generated highly polydisperse products, which further complicated the analysis of the DLS results.

\subsection{Steady-State Fluorescence of the Pyrene-Labeled NAFs and Amylopectin}

Amylopectin, NAF57, NAF20, and NAF8.3 were labeled with different amounts of 1-pyrenebutyric acid to yield Py-Amylopectin, Py-NAF57, Py-NAF20, and Py-NAF8.3, respectively. The steady-state fluorescence spectra were acquired for all samples in DMSO, and those of the Py-NAF57 and Py-NAF8.3 samples are shown in Figure $3 \mathrm{~A}$ and $3 \mathrm{~B}$, respectively. The fluorescence intensity was normalized at 376 $\mathrm{nm}$, which corresponds to the $0-0$ transition of the pyrenyl label and is set to an arbitrary value of 100 . The fluorescence spectra were acquired for samples with a pyrene concentration of $2.5 \times 10^{-6} \mathrm{M}$ that corresponded to polysaccharide concentrations lower than $26 \mathrm{mg} / \mathrm{L}$, dilute enough to avoid aggregation that would otherwise lead to intermolecular excimer formation. The fluorescence spectra show the sharp peaks of the pyrene monomer between $370 \mathrm{~nm}$ and $410 \mathrm{~nm}$ as well as the broad emission of the pyrene excimer centered at $480 \mathrm{~nm}$. More pyrene excimer was formed with increasing pyrene content due to the increased probability of encounters between the pyrenyl labels covalently attached to the polysaccharide backbone. The fluorescence spectra were further analyzed by integrating the fluorescence signal corresponding to the pyrene monomer $\left(I_{\mathrm{M}}\right)$ and excimer $\left(I_{\mathrm{E}}\right)$ to yield the $I_{\mathrm{E}} / I_{\mathrm{M}}$ ratio, which is plotted as a function of pyrene content in Figure 3C.

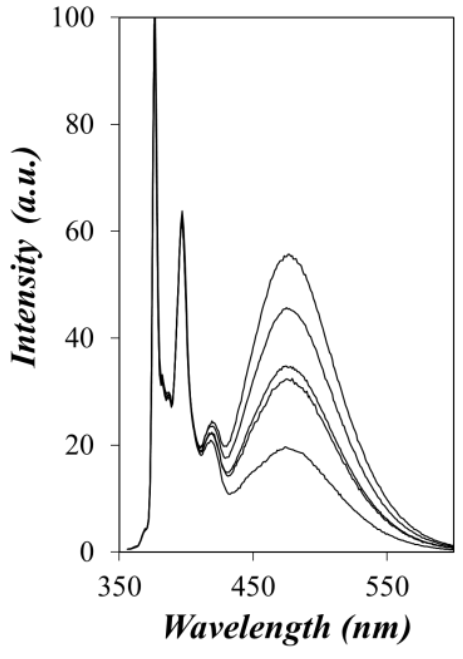

(A)

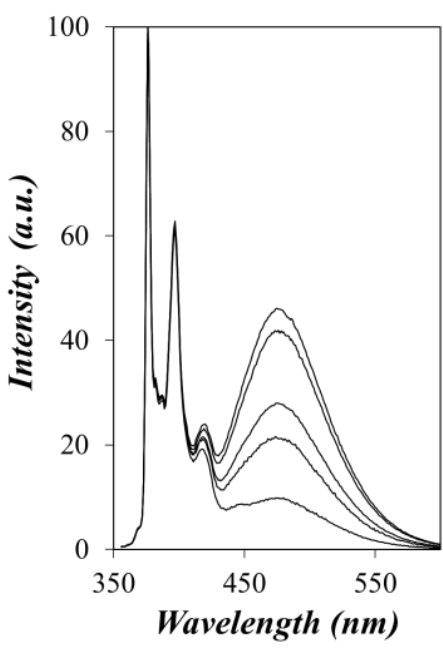

(B)

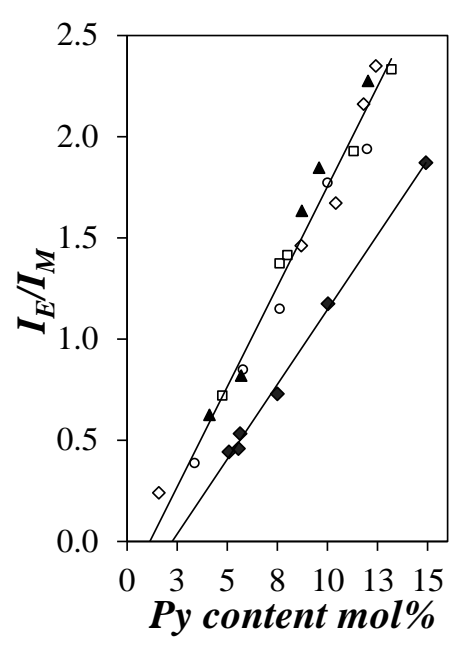

(C)

Figure 3. Fluorescence spectra of (A) Py-NAF57 and (B) Py-NAF8.3 in DMSO. From bottom to top, the pyrene content equals (A) 4.8, 7.6, 8.0, 11.3, and $13.2 \mathrm{~mol} \%$ and (B) 3.4, 5.8, 7.6, 10.0, and $12.0 \mathrm{~mol} \%$. (C) Plot of $I_{\mathrm{E}} / I_{\mathrm{M}}$ as a function of pyrene content for $(\boldsymbol{\Delta})$ Py-Amylopectin [15], ( $\square$ ) Py-NAF57, $(\diamond)$ Py-NAF20, (O) Py-NAF8.3, and $(\diamond)$ Py-Amylose [19].

When studying pyrene-labeled polymers, the $I_{\mathrm{E}} / I_{\mathrm{M}}$ ratio is typically viewed as a measure of the efficiency of pyrene excimer formation. All $I_{\mathrm{E}} / I_{\mathrm{M}}$ ratios obtained for the Py-Amylopectin, Py-NAF57, Py-NAF20, and Py-NAF8.3 samples clustered along a master line when plotted as a function of pyrene 
content, indicating similar PEF efficiencies for these four samples. This master line reflected stronger PEF efficiency with increasing pyrene content as would be expected, since a higher pyrene content promotes more pyrene-pyrene encounters, and thus more PEF. The master line also indicated that the four branched polysaccharides generated more excimer compared to the Py-Amylose samples that yielded lower $I_{\mathrm{E}} / I_{\mathrm{M}}$ ratios [19].

The stronger PEF efficiency observed for the Py-Amylopectin and Py-NAF samples was attributed to the highly branched nature of amylopectin, as depicted in Figure 1, which brought the pyrenyl labels closer to each other, thus enhancing PEF. What was remarkable in the trend shown in Figure $3 \mathrm{C}$ for Py-Amylopectin, Py-NAF57, Py-NAF20, and Py-NAF8.3 was that all $I_{\mathrm{E}} / I_{\mathrm{M}}$ ratios landed on the same master curve regardless of the size of the polysaccharide considered, which ranged from the $D_{\mathrm{h}}$ of $225 \mathrm{~nm}$ for the amylopectin sample down to the more than 20-fold smaller $D_{\mathrm{h}}$ of $8.3 \mathrm{~nm}$ for NAF8.3. This observation implied that the environment probed by an excited pyrene was the same inside these four polysaccharides. Since the reach of a 1-pyrenebutyryl label attached on the C2-hydroxyl of an AGU has been found to equal $2.3 \mathrm{~nm}$ [15], it implied that each pyrenyl label probed a same volume $\sim 4.6 \mathrm{~nm}$ in diameter, and that the local concentration of pyrenyl labels in this volume remained the same regardless of whether the polysaccharide construct was amylopectin or an NAF prepared from amylopectin.

The behavior observed in Figure $3 \mathrm{C}$ for the $I_{\mathrm{E}} / I_{\mathrm{M}}$ ratios is in perfect agreement with the Sol-CL model depicted in Figure 1. Because PEF is both short ranged (it occurs over $\sim 4.6 \mathrm{~nm}$ ) and highly efficient for the four branched polysaccharides, as seen in Figure 3C, PEF must be arising from the denser regions of the polysaccharides, which would be the clusters of helices. That PEF was unaffected by the overall size of the polysaccharide indicates that the same clusters of helices must be present in all these branched polysaccharides regardless of their actual macromolecular size. The PEF experiments, which probe a macromolecule over a $\sim 4.6 \mathrm{~nm}$ length scale for a PyBA derivative, nicely complemented the combination of DLS and $[\eta]$ measurements, which probed the macromolecule over length scales that ranged from 8.3 to $225 \mathrm{~nm}$ for the samples studied. Finally, the fact that the $I_{\mathrm{E}} / I_{\mathrm{M}}$ ratios obtained for the Py-Amylopectin [15], Py-NAF57, Py-NAF20, and Py-NAF8.3 samples were the same for a same pyrene content but remained consistently larger than the $I_{\mathrm{E}} / I_{\mathrm{M}}$ ratios obtained for Py-Amylose [19] suggests that PEF may respond to the architecture of a specific polysaccharide and could be used to differentiate between the origins of two unknown polysaccharides.

\subsection{Osmotic Pressure Experiments}

The data presented so far indicated that amylopectin and the NAFs derived from it exhibited excluded volume generated by the linear oligosaccharide segments bridging the clusters of helices, whose number decreased with decreasing $D_{\mathrm{h}}$. This feature suggested that amylopectin could be compressed if long linear polymer chains were added to the amylopectin dispersion, whose large size would prevent them from entering the amylopectin interior, thus increasing the osmotic pressure experienced by the amylopectin interior. Since the $I_{\mathrm{E}} / I_{\mathrm{M}}$ ratio is proportional to the local pyrene concentration [17], compression of Py-Amylopectin by increased osmotic pressure generated in the polysaccharide dispersion upon addition of long linear polymers should result in an increased $I_{\mathrm{E}} / I_{\mathrm{M}}$ ratio. This was indeed observed when large PEG(10K) and PEG(20K) samples were added to a dispersion of amylopectin labeled with $4.1 \mathrm{~mol} \%$ of PyBA (Py(4.1)-Amylopectin) in DMSO [15]. The large PEG samples could not penetrate the interior of $\mathrm{Py}(4.1)$-Amylopectin and a substantial increase in $I_{\mathrm{E}} / I_{\mathrm{M}}$ was observed upon increasing the polymer concentration.

While these experiments confirmed the deformability of amylopectin, they also suggested that smaller NAFs prepared from amylopectin should be harder to deform since they would have less excluded volume. To assess whether this would indeed be the case, five PEGs, namely PEG(0.2K), PEG(0.4K), PEG(2.0K), PEG(5.0K), and PEG(10K), with molecular weights of 0.2, 0.4, 2.0, 5.0, and $10 \mathrm{~K}$, respectively, were added to a dilute dispersion of Py(5.8)-NAF8.3 and Py(4.8)-NAF57 labeled with 5.8 and $4.8 \mathrm{~mol} \%$ pyrene, respectively. The $I_{\mathrm{E}} / I_{\mathrm{M}}$ ratio of the dispersions of the 
pyrene-labeled polysaccharides was monitored as a function of PEG concentration, and the results are shown in Figure 4.
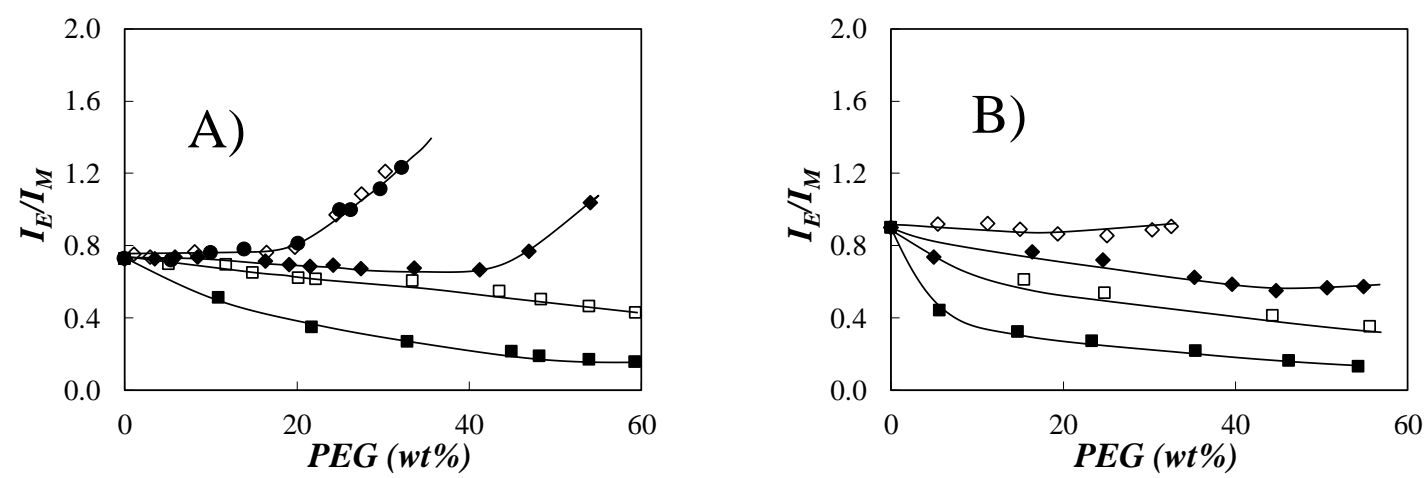

Figure 4. Plots of $I_{\mathrm{E}} / I_{\mathrm{M}}$ as a function of poly(ethylene glycol) (PEG) concentration for (A) Py(4.8)-NAF57

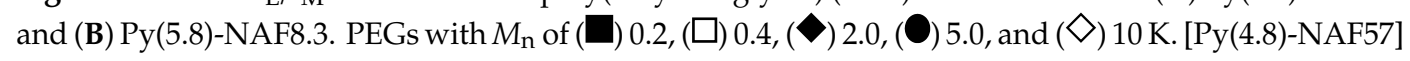
$=9.1 \mathrm{mg} / \mathrm{L},[\mathrm{Py}(5.8)-\mathrm{NAF} 8.3]=7.7 \mathrm{mg} / \mathrm{L}$.

The trends obtained by plotting $I_{\mathrm{E}} / I_{\mathrm{M}}$ as a function of PEG concentration in Figure $4 \mathrm{~A}, \mathrm{~B}$ are best explained by evoking Equation (3), which relates $I_{\mathrm{E}} / I_{\mathrm{M}}$ with the bimolecular rate constant $\left(k_{\mathrm{diff}}\right)$ for diffusion-controlled PEF and the local pyrene concentration ( $\left.[P y]_{l o c}\right)$ experienced by an excited pyrene bound to the macromolecule. Since $k_{\text {diff }}$ reflects a diffusion-controlled process, $k_{\text {diff }}$ is inversely proportional to the solution viscosity, which increases dramatically with increasing PEG concentration [15]. Furthermore, the increase in viscosity with PEG concentration is more pronounced for the longer PEGs. The decrease in $k_{\text {diff }}$ with increasing PEG concentration resulted in the decrease in the $I_{\mathrm{E}} / I_{\mathrm{M}}$ ratio with increasing PEG(0.2K) concentration for Py(4.8)-NAF57 and Py(5.8)-NAF8.3 in Figure $4 \mathrm{~A}$ and $4 \mathrm{~B}$, respectively.

$$
\frac{I_{\mathrm{E}}}{I_{\mathrm{M}}} \propto k_{\text {diff }} \times[P y]_{\mathrm{loc}}
$$

Surprisingly for a process that was diffusion controlled, the $I_{\mathrm{E}} / I_{\mathrm{M}}$ ratio decreased less with increasing concentration of larger PEGs despite the fact, that they yielded a larger solution viscosity [15]. Since $k_{\text {diff }}$ decreases with increasing PEG size at a given PEG concentration because it is inversely proportional to solution viscosity, the only explanation for the larger $I_{\mathrm{E}} / I_{\mathrm{M}}$ ratios observed with increasing PEG size was that $[P y]_{\text {loc }}$ in Equation (3) must have increased to offset the decrease in $k_{\text {diff. }}$. The increase in $[P y]_{\text {loc }}$ with increasing PEG size suggested that the larger PEGs could not penetrate the crowded interior of the NAFs, thus applying osmotic pressure to the polysaccharides. Compression of the NAFs concentrated the pyrene labels inside the NAF interior, which yielded a larger $I_{\mathrm{E}} / I_{\mathrm{M}}$ ratio. This behavior is similar to that observed for the deswelling of Py-Amylopectin with large PEG(10K) [15] or that of a swollen polymeric network that can be induced by free polymers excluded from the network [20-25].

Indeed, the effect that the osmotic pressure generated by excluded PEG chains has on poly( $\mathrm{N}$-isopropylacrylamide) (PNIPAM) microgels and macrogels has been investigated [20-24]. For a given PEG concentration, the deswelling ratio first increased with increasing molecular weight of the PEGs, before becoming independent from PEG MW when the free chains were fully excluded from the gel particles [24]. The results were actually in good agreement with the standard expression of the osmotic pressure derived by Flory and Huggins, whereby the osmotic pressure generated by the free chains depends on their volume fraction rather than their molecular weight [26]. It must be emphasized that this theory assumed that penetration of free chains into the particles could not occur. When the free chains in solution are small enough that they can diffuse into the gel particles, the chemical potential difference between the interior and exterior of the particles is partially balanced, leading to a reduction in the deswelling ratio. 
A similar effect was observed with Py-NAF57 for which the $I_{\mathrm{E}} / I_{\mathrm{M}}$ ratios obtained for Py-NAF57 with PEG(5.0K) and PEG(10K) overlapped and showed a more pronounced increase with increasing PEG concentration than that obtained with PEG(2.0K). The difference in behavior between PEG(5.0K) and PEG(2.0K) indicated that the mesh size of NAF57 corresponding to the distance separating the clusters of oligosaccharide helices was between the size of PEG(2.0K) and PEG(5.0K), since PEG(5.0K) was fully excluded from NAF57, whereas PEG(2.0K) partially penetrated it. Using the end-to-end distance $r_{\mathrm{EE}}\left(\mathrm{r}_{\mathrm{EE}}=0.119 \times \sqrt{M_{\mathrm{n}}} \times 0.707 \mathrm{~nm}\right)$ [27] for PEG chains in water, the $r_{\mathrm{EE}}$ values of 3.8 and $5.9 \mathrm{~nm}$ for PEG(2.0K) and PEG(5.0K), respectively, suggested that the mesh size of NAF57 lies between these two values at $5.0( \pm 1.0) \mathrm{nm}$. As it turns out, this NAF mesh size agrees remarkably well with a prediction made earlier that the oligosaccharide single helices inside the clusters are separated by an interhelical distance of $2.8 \mathrm{~nm}$ and that the clusters must be separated by at least $3.2 \mathrm{~nm}$ [15]. The smaller PEG $(0.2 \mathrm{~K})$ could easily fit within the helices inside and outside a cluster so that the addition of PEG $(0.2 \mathrm{~K})$ to the NAF dispersions in DMSO increased the solvent viscosity throughout the NAF interior, leading to the decrease in $I_{\mathrm{E}} / I_{\mathrm{M}}$. In contrast, the larger PEG(5.0K) and PEG(10K) were excluded from the interhelical and intercluster voids so that they remained outside the NAFs, where they increased the osmotic pressure resulting in larger $I_{\mathrm{E}} / I_{\mathrm{M}}$ ratios due to an increase in $[P y]_{\text {loc }}$.

Moreover, it is noteworthy that the compression induced by the osmotic pressure generated by the larger PEG chains would only occur for NAFs possessing sufficient excluded volume. As shown in Figure 4A, the larger Py-NAF57 sample had enough excluded volume to undergo a volume reduction in the presence of PEG(10K), whereas Py-NAF8.3 did not. In fact, for an interhelix distance $\left(d_{\mathrm{h}-\mathrm{h}}\right)$ of $\sim 2.8 \mathrm{~nm}$ predicted by fluorescence, a cluster of seven helices arranged in a hexagonal array, as shown in Figure 1, would have a diameter of $\sim 6 \mathrm{~nm}$ and a thickness of $9 \mathrm{~nm}$, equal to the thickness of crystalline lamellae found in dry amylopectin [28]. Consequently, Py-NAF8.3 with a $D_{\mathrm{h}}$ of $8.3 \mathrm{~nm}$ had a dimension comparable to that of a single cluster of helices and, as such, its compression was more limited compared to that of Py-NAF57 that had much more excluded volume as was observed experimentally in Figure 4B.

\subsection{Time-Resolved Fluorescence of Py(4.8)-NAF57}

The strong increase in $I_{\mathrm{E}} / I_{\mathrm{M}}$ observed as a function of increasing PEG(5.0K) and PEG(10K) concentration for Py(4.8)-NAF57 in Figure 4A reflected an increase in $[\mathrm{Py}]_{\mathrm{loc}}$ induced by an increase in osmotic pressure sensed by NAF57, which brought the oligosaccharide side chains bearing pyrenyl labels closer to each other. Fluorescence decays were acquired for the pyrene monomer and excimer of a $9.1 \mathrm{mg} / \mathrm{L}$ Py(4.8)-NAF57 dispersion in DMSO to which an increasing amount of PEG(5.0K) was added. The fluorescence decays were fitted according to the FBM. The fits were excellent as shown in Figure 5A,B. The parameters retrieved from the FBM analysis of the decays are listed in Tables S1-S3 in the Supplementary Materials. The FBM analysis yielded $\langle n\rangle$, which was used to calculate $N_{\text {blob }}$ according to Equation (2). $N_{\text {blob }}$ was plotted as a function of the PEG(5.0K) weight fraction in Figure 5C. $N_{\text {blob }}$ increased slightly from 17 to 19 when the PEG(5.0K) concentration increased from 0 to $20 \mathrm{wt} \%$ before increasing much more steeply from 19 to 32 when the PEG(5.0K) concentration increased from 20 to $29.3 \mathrm{wt} \%$. Interestingly, the steep increase in $N_{\text {blob }}$ for PEG(5.0K) concentrations greater than 20 wt $\%$ matched the steep increase in $I_{\mathrm{E}} / I_{\mathrm{M}}$ in Figure $4 \mathrm{~A}$, confirming that the increase in $[\mathrm{Py}]_{\text {loc }}$ probed by $I_{\mathrm{E}} / I_{\mathrm{M}}$ brought the oligosaccharide side chains closer to each other, resulting in the increase in $N_{\text {blob. }}$. The shorter interhelical distance $\left(d_{\mathrm{h}-\mathrm{h}}\right)$ separating the oligosaccharide side chains enabled more dye encounters between the pyrenyl labels, which was reflected by the increase in $N_{\text {blob }}$. 

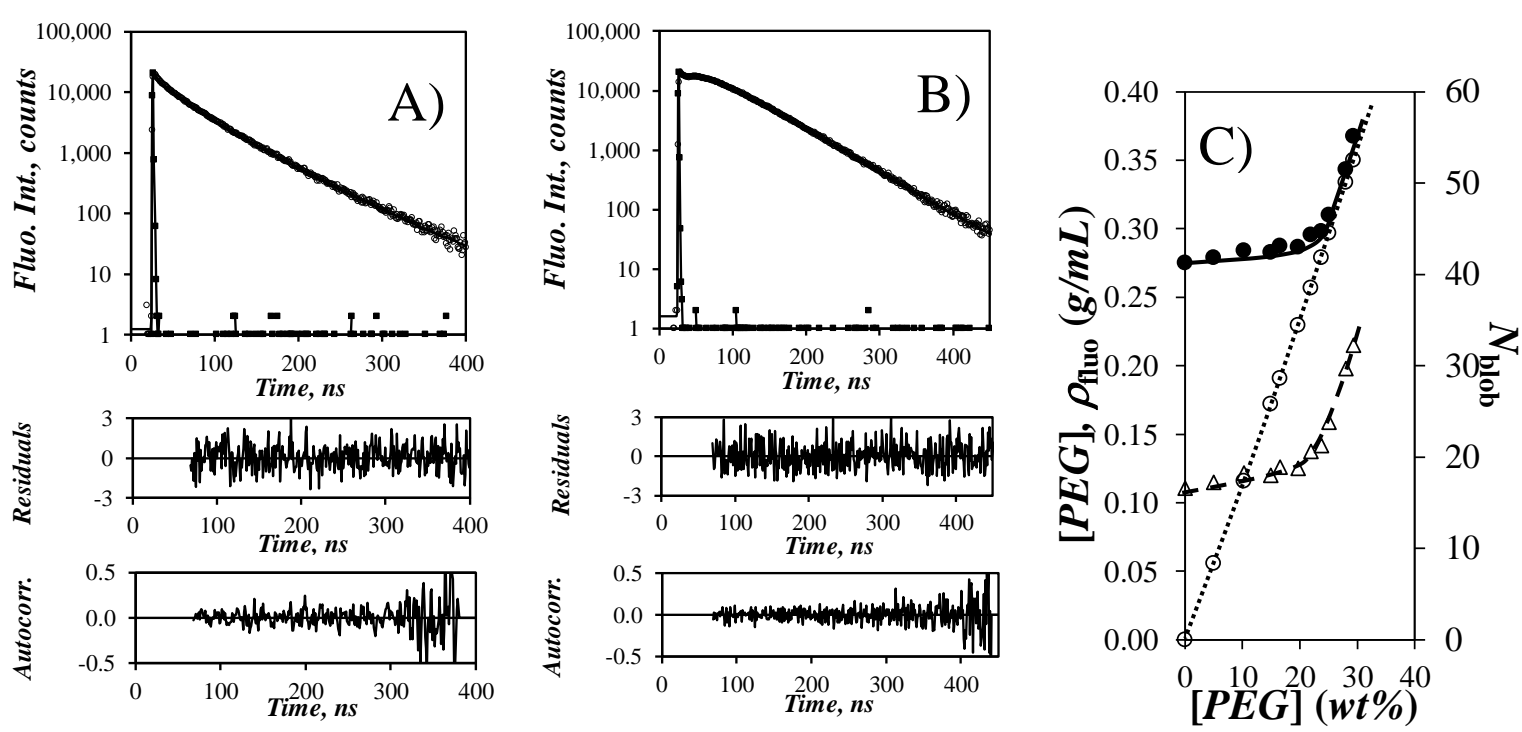

Figure 5. Fluorescence decays of the pyrene (A) monomer and (B) excimer of Py(4.8)-NAF57 in DMSO with $0.32 \mathrm{~g} / \mathrm{mL}$ PEG(5.0K). (C) Plot of $\left(\boldsymbol{\Delta}\right.$, right axis) $N_{\text {blob }},(\mathbf{O}) \operatorname{PEG}(5.0 \mathrm{~K})$ concentration, and $(\mathbf{O}) \rho_{\text {fluo }}$ as a function of PEG(5.0K) wt $\%$. [Py(4.8)-NAF57] $=9.1 \mathrm{mg} / \mathrm{L}$.

As it turns out, an earlier publication [15] established Equation (4) to relate $N_{\text {blob }}$ to $d_{\mathrm{h}-\mathrm{h}}$ for oligosaccharide single helices closely packed in a hexagonal array. In turn, $d_{\mathrm{h}-\mathrm{h}}$ can be employed to determine the density ( $\rho_{\text {fluo }}$ ) probed by the pyrenyl labels using Equation (5). $\rho_{\text {luo }}$ was plotted as a function of PEG(5.0K) concentration in Figure 5C. $\rho_{\text {fluo }}$ remained more or less constant and equal to $0.28 \mathrm{~g} / \mathrm{mL}$ until the PEG(5.0K) concentration approached $25 \mathrm{wt} \%$, at which point $\rho_{\text {fluo }}$ increased with increasing PEG(5.0K) concentration.

The concentration of PEG(5.0K) in these DMSO solutions was determined by multiplying the density of the $\mathrm{PEO}(5.0 \mathrm{~K})$ solutions in DMSO given by Equation (1) with the weight fraction of the polymer $\left(f_{\mathrm{w}}\right)$. Remarkably, the increase in $\rho_{\text {fluo }}$ coincided with the increase in PEG(5.0K) concentration, resulting in a perfect match between [PEG] and $\rho_{\text {fluo }}$ for PEG(5.0K) concentrations above $25 \mathrm{wt} \%$. The increase in $\rho_{\text {fluo }}$ from its original value of $0.28 \mathrm{~g} / \mathrm{mL}$ when the PEG $(5.0 \mathrm{~K})$ concentration approached this $\rho_{\text {fluo }}$ value is also indicative of an increase in osmotic pressure, with the local density inside the particles increasing to match the larger density generated outside the particles. This result further supports the conclusion drawn earlier that the increase in $I_{\mathrm{E}} / I_{\mathrm{M}}$ in Figure $4 \mathrm{~A}$ and $N_{\text {blob }}$ in Figure $5 \mathrm{C}$ resulted from an increase in osmotic pressure. Finally, the trend shown in Figure 5C provides further validation for the PEF-based methodology implemented earlier to determine $\rho_{\text {fluo }}$ [15].

$$
\begin{gathered}
N_{\text {blob }}=10.39+\frac{27.04}{1+\left(d_{\mathrm{h}-\mathrm{h}} / 28.06\right)^{17.34}} \\
\rho_{\text {fluo }}(\mathrm{g} / \mathrm{mL})=\frac{2.5 \times 10^{-12}}{d_{\mathrm{h}-\mathrm{h}}^{2}\left(\text { innm }^{2}\right)}
\end{gathered}
$$

\section{Conclusions}

The experiments described in this paper have consistently indicated that NAFs derived from the chemical or mechanical degradation of amylopectin have an interior that is similar to that of amylopectin as suggested by the Sol-CL model. The Sol-CL model suggests that the interior of NAFs and amylopectin is constituted of compact clusters of oligosaccharide helices linked to each other by linear oligosaccharides, which generate excluded volume. This mixture of dense and compact domains rationalizes many of the experimental observations made with amylopectin in solution and should provide a better understanding of the changes in the properties experienced by amylopectin after 
being subjected to different chemical modifications. Of particular interest was the inaccessibility of the interior of amylopectin and the NAFs derived from it to PEG chains having a $D_{\mathrm{h}}$ larger than $\sim 5.0 \mathrm{~nm}$. The deformability experiments presented in Figure 4 suggest that such large macromolecules are excluded from the interior of amylopectin and should only interact with its surface. These conclusions were further supported by using time-resolved fluorescence measurements to measure the density of the NAF57 sample as it was compressed by increasing concentrations of PEG (5.0K). The Sol-CL model appears well suited to rationalize the results described in this paper for the behavior of different polysaccharides derived from amylopectin.

Supplementary Materials: The following are available online at http://www.mdpi.com/2073-4360/12/11/2649/s1, Equations S1-S14: Equations for fluorescence decay analysis; Table S1: Parameters retrieved from the global FBM analysis with Equations (S1) and (S2) of the monomer decays of Py(5.8)-NAF57 in aerated DMSO with PEG5K., Table S2: Parameters retrieved from the global FBM analysis with Equations (S1) and (S2) of the excimer decays of Py(5.8)-NAF57 in aerated DMSO with PEG5K. $\tau_{\text {ES }}$ is fixed to equal $3.5 \mathrm{~ns}$ in the analysis, Table S3: Fractions of all

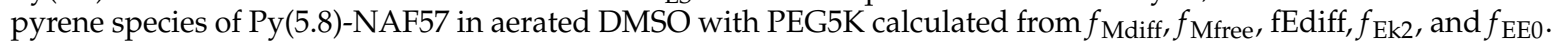

Author Contributions: Experiments and their interpretation were conducted by L.L. with supervision by J.D. who also helped during the write up of the manuscript. All authors have read and agreed to the published version of the manuscript.

Funding: This research was funded by NSERC and EcoSynthetix.

Acknowledgments: The authors wish to thank NSERC and EcoSynthetix for generous financial support. They also thank Damin Kim for conducting the experiments to obtain Equation (1).

Conflicts of Interest: The authors declare no conflict of interest.

\section{References}

1. Le Corre, D.; Bras, J.; Dufresne, A. Starch Nanoparticles: A Review. Biomacromolecules 2010, 11, 1139-1153. [CrossRef] [PubMed]

2. Delcour, J.A.; Bruneel, C.; Derde, L.J.; Gomand, S.V.; Pareyt, B.; Putseys, J.A.; Wilderjans, E.; Lamberts, L. Fate of Starch in Food Processing: From Raw Materials to Final Food Products. Ann. Rev. Food Sci. Technol. 2010, 1, 87-111. [CrossRef] [PubMed]

3. Giezen Franciscus, E.; Jongboom Remigius Oene, J.; Feil, H.; Gotlieb Kornelis, F.; Boersma, A. Biopolymer Nanoparticles. U.S. Patent US 6677386 B1, 13 January 2004.

4. Hancock, R.D.; Tarbet, B.J. The Other Double Helix-The Fascinating Chemistry of Starch. J. Chem. Educ. 2000, 77, 988-992. [CrossRef]

5. Campos, E.; Branquinho, J.; Carreira, A.S.; Carvalho, A.; Coimbra, P.; Ferreira, P.; Gil, M.H. Designing Polymeric Microparticles for Biomedical and Industrial Applications. Eur. Polym. J. 2013, 49, 2005-2021. [CrossRef]

6. Rayner, M.; Marku, D.; Eriksson, M.; Sjöö, M.; Dejmek, P.; Wahlgren, M. Biomass-Based Particles for the Formulation of Pickering Type Emulsions in Food and Topical Applications. Colloids Surf. A 2014, 458, 48-62. [CrossRef]

7. Pei, X.; Tan, Y.; Xu, K.; Liu, C.; Lu, C.; Wang, P. Pickering Polymerization of Styrene Stabilized by Starch-Based Nanospheres. Polym. Chem. 2016, 7, 3325-3333. [CrossRef]

8. Ye, F.; Miao, M.; Lu, K.; Jiang, B.; Li, X.; Cui, S.W. Structure and Physicochemical Properties for Modified Starch-Based Nanoparticle from Different Maize Varieties. Food Hydrocoll. 2017, 67, 37-44. [CrossRef]

9. Simi, C.K.; Emilia Abraham, T. Hydrophobic Grafted and Cross-Linked Starch Nanoparticles for Drug Delivery. Bioprocess Biosyst. Eng. 2007, 30, 173-180. [CrossRef]

10. Pei, X.; Zhai, K.; Tan, Y.; Xu, K.; Lu, C.; Wang, P.; Wang, T.; Chen, C.; Tao, Y.; Dai, L.; et al. Synthesis of Monodisperse Starch-Polystyrene Core-Shell Nanoparticles via Seeded Emulsion Polymerization without Stabilizer. Polymer 2017, 108, 78-86. [CrossRef]

11. Kang, B.G.; Yoon, S.H.; Lee, S.H.; Yie, J.E.; Yoon, B.S.; Suh, M.H. Studies on the Physical Properties of Modified Starch-Filled HDPE Film. J. Appl. Polym. Sci. 1996, 60, 1977-1984. [CrossRef]

12. Kim, D.; Amos, R.; Gauthier, M.; Duhamel, J. Applications of Pyrene Fluorescence to the Characterization of Hydrophobically Modified Starch Nanoparticles. Langmuir 2018, 34, 8611-8621. [CrossRef] [PubMed] 
13. Kim, D.; Amos, R.C.; Gauthier, M.; Duhamel, J. Assemblies of Hydrophobically Modified Starch Nanoparticles Probed by Surface Tension and Pyrene Fluorescence. In ACS Symposium Series Nanoparticles and Molecular Assemblies; Nagarajan, R., Ed.; American Chemical Society: Washington, DC, USA, 2020.

14. Wildi, R.H.; Van Egdob, E.; Bloembergen, S. Process for Producing Biopolymer Nanoparticles. U.S. Patent PCT/US2007/075901, 21 February 2008.

15. Li, L.; Kim, D.; Zhai, X.; Duhamel, J. A Pyrene Excimer Fluorescence (PEF) Study of the Interior of Amylopectinin Dilute Solution. Macromolecules 2020, 53, 6850-6860. [CrossRef]

16. Winnik, F.M. Photophysics of Preassociated Pyrenes in Aqueous Polymer Solutions and in Other Organized Media. Chem. Rev. 1993, 93, 587-614. [CrossRef]

17. Cuniberti, C.; Perico, A. Intramolecular Excimer Formation in Polymers Pyrene-Labeled Polyvinylacetate. Eur. Polym. J. 1980, 16, 887-893. [CrossRef]

18. Millard, M.M.; Dintzis, F.R.; Willett, J.J.; Klavons, J.A. Light Scattering Molecular Weights and Intrinsic Viscosities of Processed Waxy Maize Starches in 90\% Dimethyl Sulfoxide and $\mathrm{H}_{2} \mathrm{O}$. Cereal Chem. 1997, 74, 687-691. [CrossRef]

19. Li, L.; Duhamel, J. Conformation of Pyrene-Labeled Amylose in DMSO Characterized with the Fluorescence Blob Model. Macromolecules 2016, 49, 7965-7974. [CrossRef]

20. Bastide, J.; Candau, S.; Leibler, L. Osmotic De-Swelling of Gels by Polymer-Solutions. Macromolecules 1981, 14, 719-726. [CrossRef]

21. Inomata, H.; Nagahama, K.; Saito, S. Measurement and Correlation of the Swelling Pressure of N-Isopropylacrylamide Gel. Macromolecules 1994, 27, 6459-6464. [CrossRef]

22. Nagahama, K.; Inomata, H.; Saito, S. Measurement of Osmotic-Pressure in Aqueous-Solutions of Poly (Ethylene Glycol) and Poly (N-Isopropylacrylamide). Fluid Phase Equilib. 1994, 96, 203-214. [CrossRef]

23. Ishidao, T.; Akagi, M.; Sugimoto, H.; Iwai, Y.; Arai, Y. Swelling Behaviors of Poly (N-Isopropylacrylamide) Gel in Poly (Ethylene Glycol)—Water Mixtures. Macromolecules 1993, 26, 7361-7362. [CrossRef]

24. Ishidao, T.; Akagi, M.; Sugimoto, H.; Onoue, Y.; Iwai, Y.; Arai, Y. Swelling Equilibria of Poly (N-Isopropylacrylamide) Gel in Aqueous Polymer-Solutions. Fluid Phase Equilib. 1995, 104, 119-129. [CrossRef]

25. Saunders, B.R.; Vincent, B. Thermal and Osmotic Deswelling of Poly (NIPAM) Microgel Particles. J. Chem. Soc. Faraday Trans. 1996, 92, 3385-3389. [CrossRef]

26. Flory, P.J. Principles of Polymer Chemistry; Cornell University Press: Ithaca, NY, USA, 1953; p. 672.

27. Pattanyek, S.K.; Juvekar, V.A. Prediction of Adsorption of Nonionic Polymers from Aqueous Solutions to Solid Surfaces. Macromolecules 2002, 35, 9574-9585. [CrossRef]

28. Perez, S.; Bertoft, E. The Molecular Structures of Starch Components and Their Contribution to the Architecture of Starch Granules: A Comprehensive Review. Starch Stärke 2010, 62, 389-420. [CrossRef]

Publisher's Note: MDPI stays neutral with regard to jurisdictional claims in published maps and institutional affiliations.

(C) 2020 by the authors. Licensee MDPI, Basel, Switzerland. This article is an open access article distributed under the terms and conditions of the Creative Commons Attribution (CC BY) license (http://creativecommons.org/licenses/by/4.0/). 